\title{
A NEW FINGER-VEIN RECOGNITION SYSTEM USING THE COMPLETE LOCAL BINARY PATTERN AND THE PHASE ONLY CORRELATION
}

\author{
Ahmed A. Mustafa \\ Department of Computer Science \\ University of Duhok, \\ Duhok, Iraq \\ ahmed.aabaker@uod.ac \\ Ahmed AK. Tahir \\ Department of Computer Science \\ University of Duhok \\ Duhok, Iraq \\ ahmdi@uod.ac
}

Submitted: Apr, 10,2021 Revised: Jun, 13, 2021 Accepted: Jun, 23, 2021

\begin{abstract}
A new system for finger-vein recognition is proposed based on the Complete Local Binary pattern (CLBP) as a feature extractor and the Phase Only Correlation (POC) for post-processing alignment and for speeding up the system. The CLBP produces three components of image descriptors and thus holds more details compared to the previous methods such as the Local Binary Pattern (LBP), the Local Directional Pattern (LDP), the Local Line Binary Pattern (LLBP), the Repeated Line Tracking (RLT), the Maximum Curvature (MC) and the Wide Line Detector (WLD). In the proposed system, POC is used for two purposes. First, to increase the performance of the system the alignment between the CLBP components of the test image and the enrolled CLBP components are performed. Second, to speed up the matching stage, a portion of the enrolled images is excluded that are highly misaligned with the test image from the Hamming Distance (HD) measure competition in the matching stage. To make the system more secure against attacks targeting personal information, only CLBP components are enrolled in the system and the alignment process POC is implemented on these components without the need to original images. For image pre-processing a novel scheme of preprocessing methods is adopted including finger-vein localization, alignment, and the Region-Of-Interest (ROI) extraction and enhancement. Two databases, UTFVP and SDUMLA-HMT, are used to evaluate the performance of the system. The results have shown that the values for the Identification Recognition Rate (IRR) and the Equal Error Rate (EER) are respectively $(99.66 \%)$ and $(0.139)$ for the UTFVP database and $(98.95 \%$, and $0.53 \%)$ for SDUMLA-HMT database. These results are competitive compared to those achieved by the state-of-art systems.
\end{abstract}

Keywords: Biometrics, finger vein, feature extraction, phase only correlation.

\section{INTRODUCTION}

The field of automatic person identification that is based on human biometric recognition is becoming a necessary approach for security purpose. An extensive number of biometric security systems using iris, fingerprints, face, finger-veins, palm-vein, voice and signature, have already been developed and used successfully in various applications, such as e-commerce, cross point checking, automobile security and employee attendance tracking, [1-4], "in press" 
[5]. However, a direct comparison between different human traits is difficult, due to the reason that each biometric technique has its own merits and shortcoming. The success of biometric recognition system depends on the strength of the biometric trait and the required level of security for which the system is designed, [6-10].

Finger-vein recognition is relatively a recent but very effective biometric trait compared to other human traits. It has a unique pattern of blood vessel for each person and it has several advantages that make it more secure and acceptable compared to other human biometrics. First, finger-veins are located under the surface of the skin; therefore it will not be affected by skin damage. For instance, finger-vein provides more secure and safety compared to fingerprints. Second, finger-veins are living bodies so they cannot be cheated, $[11,12]$. Third, finger-vein is more acceptable compared to iris and is less affected by environment and weather conditions such as sweaty, dryness, humidity and high or low temperature. However, it may have several disadvantages, if not handled properly. First, the image of finger-vein contains undesirable black background and affected by the uneven illumination conditions. Second, the images of the same fingerveins may suffer severe misalignment due to the unfixed position of the finger on the acquisition devices. Third, some diseases such as, diabetes, atherosclerosis, hypertension, etc., may affect it.

In this paper, a new finger-vein recognition system for person identification is proposed. The system is based on using the technique of Complete Local Binary pattern (CLBP) as feature extraction and the technique of Phase Only Correlation (POC) for image alignment and speeding up the matching stage.

The main contribution of this work can be summarized as: First, the technique of CLBP is used for the first time for finger vein recognition. It has been used previously for face recognition and texture classification. Second, the technique of POC is used to achieve image alignment and to speed up the stage of image matching.

The remainder of this paper is organized as follows: Section II introduces the related work. Sections III-VI present the layout and the details of the proposed system. Sections VII and VIII presents system implementation and evaluation. Finally, in section IX the conclusions are given.

\section{RELATED WORKS}

The first system for finger-vein recognition was developed by Hitachi Central Research Laboratory in 2004, [13]. It was based on Repetitive Line Tracking (RLT) method. The system achieved an Equal Error rate (EER) of $(0.145 \%)$ using database taken from 678 persons. In 2007, the same research group developed another system using the Maximum Curvature method (MC) for feature extraction, they achieved EER of $(0.145 \%)$, [14]. In MC method, the local maximum curvatures are calculated in four directions (horizontally, vertically and diagonally), and the center points on the veins are obtained from the curvatures and connected to each other to form the final vein pattern network. Song and others, [15] introduced a system with modified MC by considering the curvatures in all directions. However, their system suffered from one major drawback by using the original image as a reference template, which makes the system vulnerable to the attacks targeting on personal information.

In 2009, the technique of LBP and its modified versions were used. For instance, Lee and others, [16] developed a system using local binary pattern (LBP) technique for feature extraction and a combination of Lee masks and affine transform for preprocessing. They achieved EER of 0.08\%. Later in 2010, the 
same authors, [17] improved this EER to $0.049 \%$ by using the weighted local binary pattern code based on a Support Vector Machine (SVM). Park, [18], also used the technique of LBP combined with wavelet transform for extracting the local and global features. Park's system achieved EER of $0.011 \%$. In 2011, Rosdi and others, [19], modified the technique of LBP to Local Line Binary Pattern (LLBP) as an aim to improve the performance and the speed of the system. For pre-processing, Rosdi team used image binarization followed by Otsu's threshold method and Gaussian high-pass filter for ROI extraction and enhancement and finally the technique of Phase Only Correlation (POC) was used for ROI alignment. Rosdi and others' system achieved EER of $1.78 \%$ with a database of 2040 images. In 2011, Lee and others [1] compared the feature extraction method of Local Derivative Pattern (LDP) to binarization and LBP for the accuracy and speed. They concluded that LDP achieved better results than the methods of Binarization and LBP.

In 2010, Huang and others, [20] introduced the Wide Line detector (WLD), which is based on comparing the pixel intensities within a circular region to a predefined threshold for binarization. They achieved an EER of 2.86\%. In 2013, [21] enhanced the LLBP to Polydirectional Local Line Binary Pattern (PLLBP) by coding the features in vertical, horizontal, diagonal and anti-diagonal directions. For pre-processing, they used Hough transform, multi-resolution analysis and CLAHE. They achieved an Identification Recognition Rate (IRR) of 99.21 using SDUMLA-HMT database. Later in 2017, [22] introduced a new finger-vein system that was based on Customized Local Line Binary Pattern (CLLBP) which considers the coding in eight directions. Liu and others' system achieved EER of $0.055 \%$ using Hong Kong Polytechnic University (HKPU) database.

Other finger-vein systems, [3,23,24] that are based on Gabor filter for feature extraction were developed. Despite different methods of pre-processing and matching were used, these systems achieved IRR above 98\%. Peng and others, [25] developed a system with RLT feature extraction and a combination of filtering, Hough transform and histogram projection and a Non-Local Means algorithm (NLM) for ROI extraction. They achieved EER of 5.5\% using Hong Kong Polytechnic University Database. However, the system was slow due to the use of Hough transform and the NLM algorithm.

Kaur and others, [26] also used RLT and Gabor filters for extracting fingervein patterns. For pre-processing, they used image binarizing and Sobel edge detector for ROI extraction. Yahaya and others, [27] combined MC method with a Directional-based feature extraction method (MCDF). For pre-processing, they used boundary detection followed by rotation correction, binarization and thinning. They achieved IRR of $99.17 \%$ using SDUMLA-HMT. Brindha, [28] extracted minutiae points as and for pre-processing he used Gabor filters to remove the noise and preserve true ridge/valley structures and two morphological operations for ROI extraction. Brindha's system achieved EER of $8.1 \%$, using only a part of SDUMLA-HMT database.

Syarif and others, [29] used a combination of the Enhanced Maximum Curvature (EMC) method and the Histogram of Oriented Gradient (HOG) for feature extraction. They achieved EER of 0.14\% using SDUMLA-HMT database. Lu and others, [30] developed a system by deriving the Pyramid Histogram of Double Competitive Pattern (PHDCP) from a bank of Gabor filters. They achieved EER of $(0.33 \%)$ and $(2.91 \%)$ for MMCBNU_6000 and UTFVP databases respectively. Meng and others, [31] used the dense Scale Invariant Feature Transform (dense SIFT) for feature extraction and a pixel-based displacements as a discriminative measure for image matching. They achieved an EER of $(2.68 \%)$ using SDUMLAHMT database. Wang and others, [32] modified the Weber Local Descriptor (WLD) to a Directional Curvature Gabor Weber Local Descriptor (DCGWLD) by adding the 
directional information to the extracted features. They achieved EERs of $1.41 \%$ and $2.69 \%$ using SDUMLA-HMT and PolyU databases respectively.

Recently, the approach of Convolutional neural networks (CNNs) has emerged and many CNN based systems were developed. Hong and others [3] developed a system using VGG Net-16 and achieved EER of (3.906) using SDUMLA-HMT database. Das and others, [4] used CNN for finger-vein recognition and obtained IRR of (97.48 and 95.56) for SDUMLA-HMT and UTFVP databases respectively. Tang and others, [33] introduced a CNN based system using three datasets, SDUMLA-HMT, MMCBNU_6000 and FV-USM. However, CNN based systems require to be retrained after each update of database. In addition, it require a good selection of the CNN parameters in order to perform well, $[34,35]$.

\section{PROPOSED SYSTEM}

The proposed system includes three major modules, pre-processing, feature extraction and matching. Figure 1 shows the block-diagram of the proposed system. The system can be operated in two modes, enrolment and testing. In the enrollment mode, the extracted features are stored as a database to be used for comparison in the testing mode. In the testing mode, the features are extracted from the original finger vein image and aligned and compared with the enrolled features. In the proposed system, The POC function is used for two purposes, image alignment and a priori matching operation by nominating only those images that shows small $\mathrm{x}-\mathrm{y}$ displacement to the process of final matching by HD measure. The image descriptors rather than the original image are involved the process of POC. This will increase the security against any attack on the person information within the system. The details of these modules are given in the following sections.

\section{A. Pre-Processing}

Pre-processing is an important stage in designing finger-vein recognition system and must be handled attentively. It has been mentioned by [36], that an optimal pre-processing must result in an efficient image descriptor. Many schemes of pre-processing have been developed and have been proven to achieve a significant improvement in the performance of finger-vein recognition systems, [16, $19,21,24,27,30,31,37,38]$. The most recent of these schemes was suggested by [38] and was proven to be effective for improving the system performance even with different methods of feature extraction. This scheme of pre-processing includes the detection of finger-vein boundary using the two masks followed by rotation correction for alignment and ROI extraction and finally the methods of ROI enhancement which includes the CLAHE, median filter and finally the modified high pass Gaussian filter. In this paper, this scheme is adopted except, the modified Gaussian high pass filter is not used. Instead, the proposed method of feature extraction performs an equivalent technique inclusively via one of its components, which is called the magnitude component. 
Int. J.Adv.Sig.Img.Sci, Vol. 7, No. 1, 2021

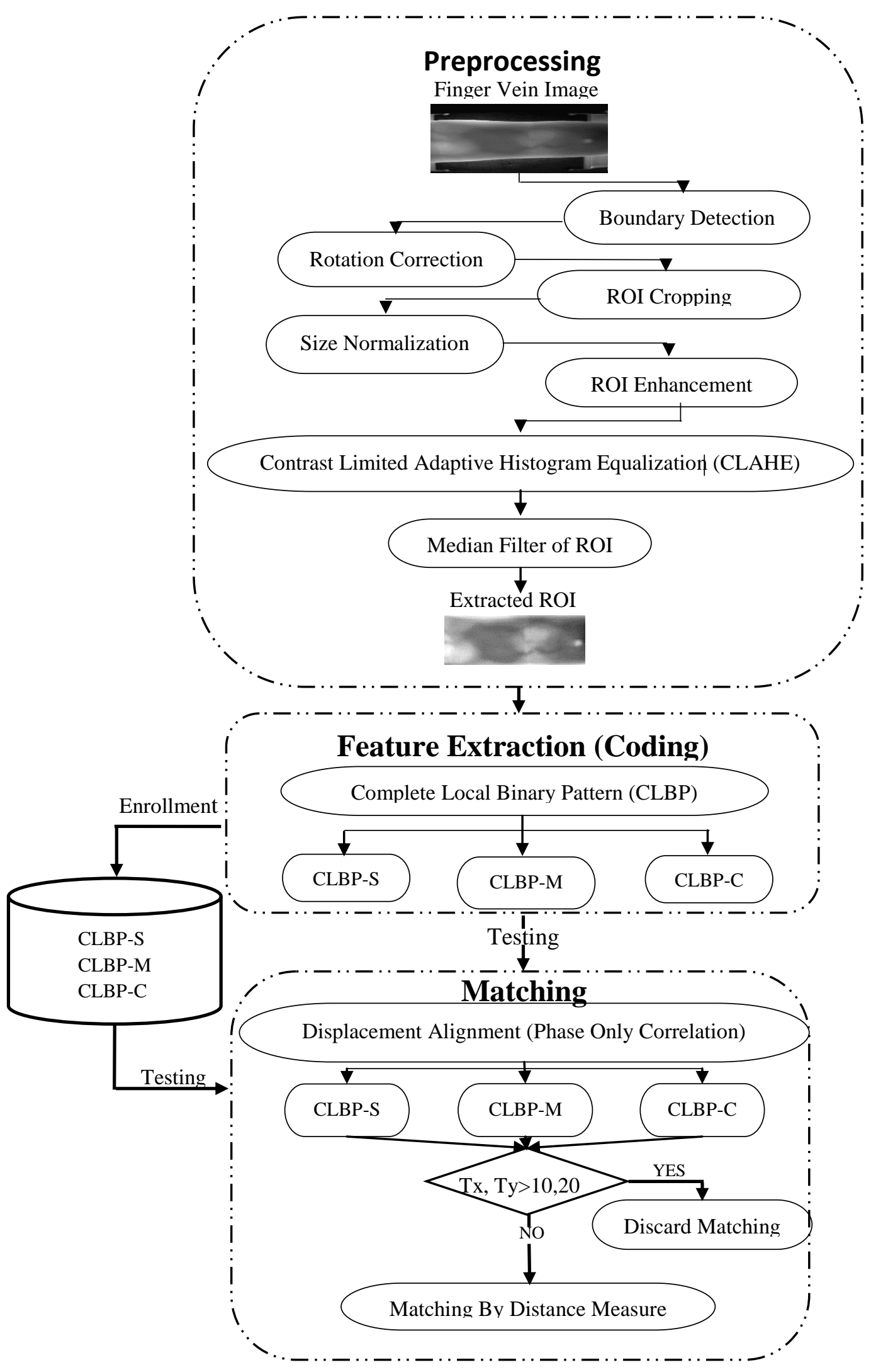

Fig. 1 The block-diagram of the proposed system for finger vein recognition 


\section{B. Feature Extraction; Complete Local Binary Pattern}

The Completed Local Binary Pattern (CLBP) descriptor which is an a modified version of LBP descriptor was modified for the first time by [39] and used by [40,41] for texture classification and by [42] for improving the performance of face recognition system. It was also used along with the curvelet transform by [43] for facial expression representation. However, for the finger-vein recognition, to the author's knowledge, has not been used. In the proposed system, this method is used for feature extraction. The CLBP descriptor is constructed from the decomposition of two complementary components from the local variance image. The magnitude and the sign of the components which are denoted by $m_{p}$ and $s_{p}$ are calculated according to $[39,41]$ by the following equations:

$$
\begin{aligned}
& s_{p}=s\left(i_{p}-i_{c}\right) \\
& m_{p}=\left|i_{p}-i_{c}\right|
\end{aligned}
$$

Then the two operators CLBP_Magnitude (CLBP_M) and CLBP_Sign (CLBP_S) are calculated from $\mathrm{m}_{\mathrm{p}}$ and $\mathrm{s}_{\mathrm{p}}$ according to $[39,41]$ using the formula below:

$$
\begin{gathered}
\text { CLBP_M }_{\mathrm{P}, \mathrm{R}}=\sum_{p=0}^{p-1} 2^{p} t\left(m_{p}, c\right), t\left(m_{p}, c\right)\left\{\begin{array}{cc}
1 & \text { if }\left|i_{p}-i_{c}\right| \geq c \\
\text { or } & \text { if }\left|i_{p}-i_{c}\right|<c
\end{array}\right. \\
\text { CLBP_S }_{P, R}=\sum_{p=0}^{p-1} 2^{p} s\left(i_{p}-i_{c}\right), s_{p}=\left\{\begin{array}{cc}
1 & \text { if } i_{p} \geq i_{c} \\
0 & \text { or } \\
0 & \text { if } i_{p}<i_{c}
\end{array}\right.
\end{gathered}
$$

where, $i_{c}$ is the intensity of the central pixel, $i_{p}(p=0,1, \ldots, P-1)$ is the intensity of the adjacent pixels, $\mathrm{p}$ denotes the number of neighbors and $\mathrm{R}$ is the radius of the circle enclosing the neighboring pixels, $c$ is the mean value of $m_{p}$ in the whole image.

CLBP_S operator is the same as LBP preserves the information about image local features and it is robust to illumination changes. CLBP_M operator is the measure of the magnitude local differences and adds further discriminant information, it is equivalent to the modified Gaussian high pass filter. A new operator called CLBP-Center CLBP_C, which also add discriminant information concerning the image local intensity, can be constructed from the intensity of the central pixel and the mean intensity of the whole image as shown below:

$$
C L B P_{-} C_{P, R}=t\left(i_{p}, C_{I}\right), t\left(i_{c}, C_{I}\right)=\left\{\begin{array}{c}
1 \quad \text { if } i_{p} \geq C_{I} \\
\text { or } \\
0 \quad \text { if } i_{p}<C_{I}
\end{array}\right.
$$

According to $[39,41]$,ip is the intensity of the processed pixel and $C_{I}$ is the average intensity of the whole image. To improve the recognition accuracy, Guo and others combined the three operators,CLBP_M, CLBP_S and (CLBP_C).

In the proposed system, the three CLBP components, CLBP_M, CLBP_S and CLBP_C are used as extracted features for finger-vein recognition. For an illustration of calculating the CLBP operators, (see Figure. 2).

The results of applying CLBP and obtaining the three image descriptors CLBP_M, CLBP_S and CLBP_C are shown in Figure 3. Each of the three components reveals a unique type of details. The CLBP_M component is equivalent to a pre-processed image including the modified Gaussian high pass filter, CLBP-S is equivalent to LBP image descriptor and CLBP-C details concerning the vein net. 


\begin{tabular}{|c|c|c|c|c|c|c|}
\hline 31 & 90 & 102 & & 0 & 1 & 1 \\
\hline 79 & 34 & 20 & & 1 & & 0 \\
\hline 12 & 33 & 2 & & 0 & 0 & 0 \\
\hline & & & & \multicolumn{3}{|c|}{ CLBP_S $=10000110$} \\
\hline 3 & 56 & 68 & \multirow{3}{*}{$\mathrm{C}=29$} & 0 & 1 & 1 \\
\hline 36 & & 14 & & 1 & & 0 \\
\hline 22 & 1 & 32 & & 0 & 0 & 1 \\
\hline
\end{tabular}

Fig. $2(3 \times 3)$ sample pattern shows how CLBP_S and CLBP_M are calculated.

\begin{tabular}{|c|c|c|c|}
\hline $\begin{array}{c}\text { CLBP } \\
\text { Components }\end{array}$ & \multicolumn{3}{|c|}{ CLBP of UTFVP Images } \\
\hline Original & & & \\
\hline CLBP_M & DAc 1 - & & \\
\hline CLBP_S & 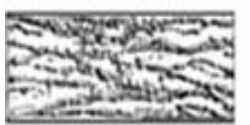 & 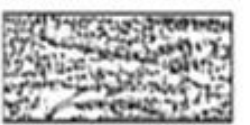 & 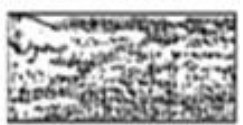 \\
\hline CLBP_C & indsis & 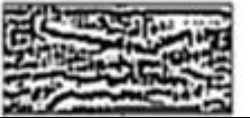 & 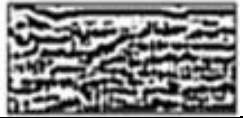 \\
\hline $\begin{array}{c}\text { CLBP } \\
\text { Components }\end{array}$ & CLBP of & SDUMI & Images \\
\hline Original & & & \\
\hline CLBP_M & 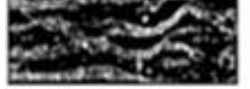 & 25 & 대에 \\
\hline CLBP_S & $x^{2}=2$ - & 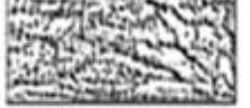 & $\begin{array}{l}3040 \\
34 c\end{array}$ \\
\hline CLBP_C & 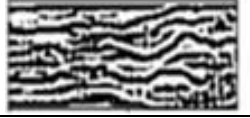 & H. & Fris \\
\hline
\end{tabular}

Fig. 3 CLBP image descriptors for two samples of finger-vein images taken from UTFVP and SDUMLA-HMT datasets. 


\section{Matching}

In the proposed system, the matching stage includes two parts, the use of POC and image comparison by Hamming Distance Measure (HD). The technique of POC is used for two purposes, increasing the recognition rate by implementing image alignment and speeding up the matching stage by excluding a portion of the enrolled images that show large $x-y$ displacement with the test image from the process of matching by HD measure. The HD measure is used because it is suitable for comparing two images on the pixel-by-pixel basis. Other classifiers such as Artificial Neural Network (ANN) and the Support Vector Machin (SVM) are more convenient when the features are in the form of one-dimensional vector. The following subsections illustrate, in details, the use and implementation of POC and the HD computation.

\section{C.1. Image Alignment by the Phase Only Correlation (POC)}

One of the problems that faces the finger vein recognition is the variability in the finger position and orientation during the vein detection. This problem causes the images of the same vein to be misaligned leading to a deficiency in the finger vein recognition performance. The technique of the Phase Only Correlation (POC) is used to calculate the displacements in $\mathrm{x}$ and $\mathrm{y}$ directions between two images. The technique computes the normalized cross spectrum between the Fourier transform of two images and inverses it back to spatial domain. The coordinates of the pixel having the maximum peak value represents the displacement in $\mathrm{x}$ and $\mathrm{y}$ axes between the two images. These displacements are used to align the two images. The POC technique was used, for the first time by [44] for image registration in computer vision applications. Later, it was used by [45-47] as a matching metric for fingerprint, palmprint and finger vein recognition.

In this paper, the POC function is used for two purposes. First, for securing the person information against any attack the process of alignment between one of the CLBP components of the test image and the enrolled CLBP_S images is implemented. Only CLBP_S is used because it holds more information, but the extracted $\mathrm{x}$ and $\mathrm{y}$ displacements are applied to all the three CLBP components. The use of CLBP_S component instead of the original image will guarantee the system security. Second, for speeding up the matching stage, the $\mathrm{x}$ and y displacements are utilized as measures for initial matching. In order to save computation time, threshold values for the $\mathrm{x}$ and $\mathrm{y}$ displacements are defined and only the enrolled images that pass these threshold values will be candidates for the HD measurement and final matching In POC, the two images are Fourier transformed, and then the cross phase spectrum is calculated, and transformed by inverse Fourier transform. Finally, the $\mathrm{x}$ and $\mathrm{y}$ coordinates of the maximum peak are used for image alignment, [45-47]. Figure 4 shows the procedures of calculating the POC function. 
Image 1
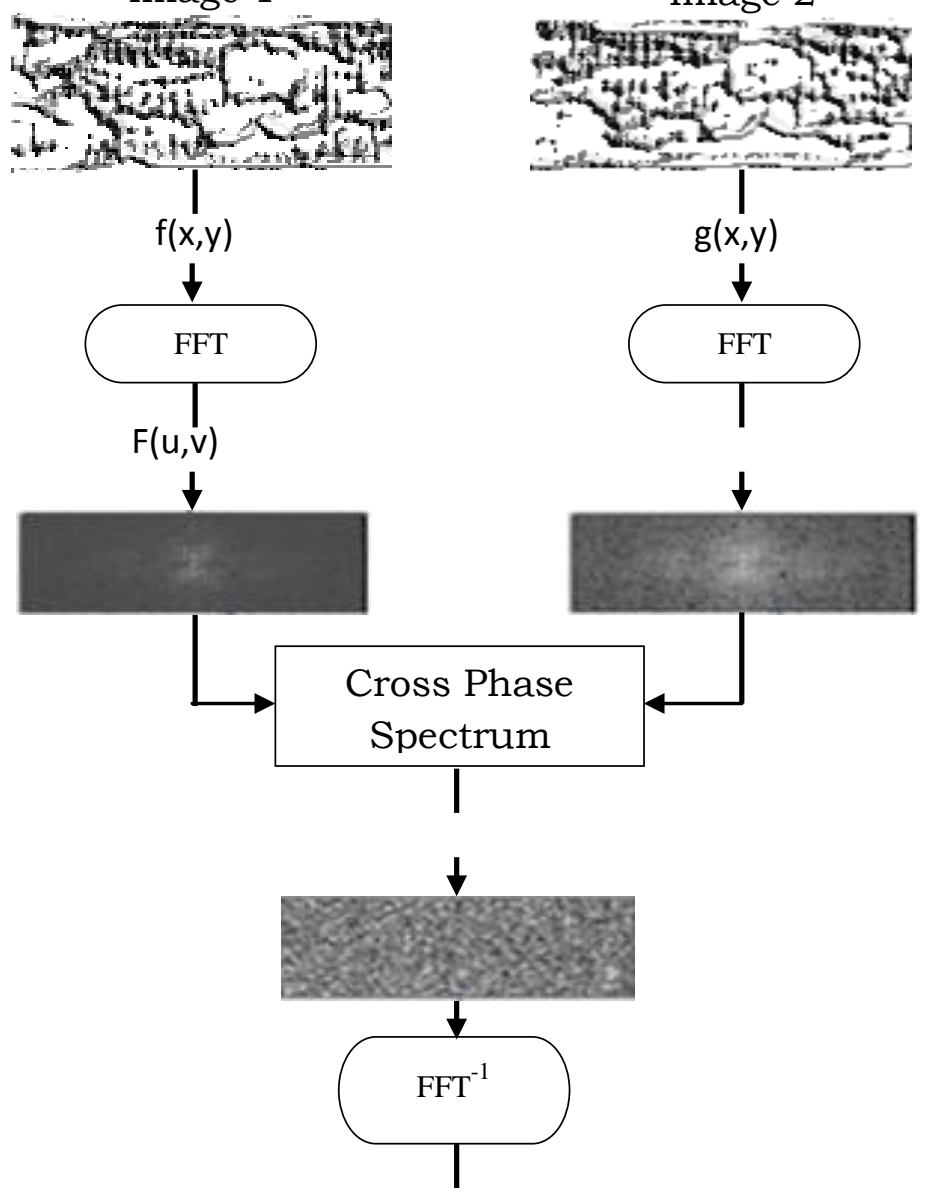

$\downarrow$

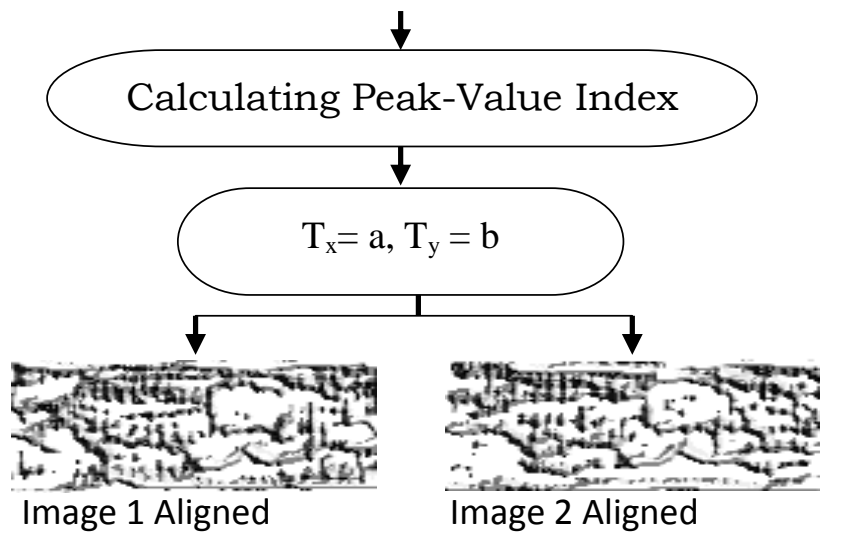

Fig. 4 The procedures of implementing POC

\section{C.2 Matching Metrics}

In image matching, HD is used to calculate the similarity between the CLBP components of test image and the CLBP components of the enrolled database. As mentioned, the distance is measured between all three CLBP components of the test images and the enrolled CLBP components that pass the displacement threshold test. 


\section{IMPLEMENTATION AND RESULTS}

The proposed system with CLBP is implemented with and without POC using two types of datasets. The details are given in the following subsections.

\section{A. Datasets}

Two types of databases are used, the University of Twente Finger Vascular Pattern (UTFVP) and the Shandong University Machine Learning and Applications - Homologous Multi-modal Traits SDUMLA-HMT, [48, 49]. The images of these two databases are of different quality. The UTFVP images are known to be misaligned, but of better contrast quality compared to SDUMLA-HMT images, [50]. Therefore, they represent good environment for testing the efficiency and adaptability of the systems. Table 1 shows the details of the two databases and Figure 5 shows some samples of finger-vein images taken from UTFVP and SDUMLA-HMT datasets.

TABLE 1 Details of the UTFVP and SDUMLA-HMT datasets

\begin{tabular}{|c|c|c|c|c|c|}
\hline $\begin{array}{c}\text { Database } \\
\text { Type }\end{array}$ & $\begin{array}{c}\text { No. Of } \\
\text { Subjects }\end{array}$ & $\begin{array}{c}\text { No. Of } \\
\text { Images }\end{array}$ & $\begin{array}{c}\text { No. Of } \\
\text { Finger/Subject }\end{array}$ & $\begin{array}{c}\text { No. Of } \\
\text { Image/Subject }\end{array}$ & $\begin{array}{c}\text { Image } \\
\text { Size }\end{array}$ \\
\hline UTFVP & 60 & 1440 & $\begin{array}{c}6 \text { for both hands } \\
\text { (middle, index } \\
\text { ring) }\end{array}$ & 6 & $672 \times$ \\
380 & & $320 \mathrm{x}$ \\
\hline $\begin{array}{c}\text { SDUMLA } \\
\text {-HMT }\end{array}$ & 106 & 3816 & $\begin{array}{c}\text { 6 for both hands } \\
\text { (middle, index } \\
\text { ring) }\end{array}$ & 6 & 240 \\
\hline
\end{tabular}

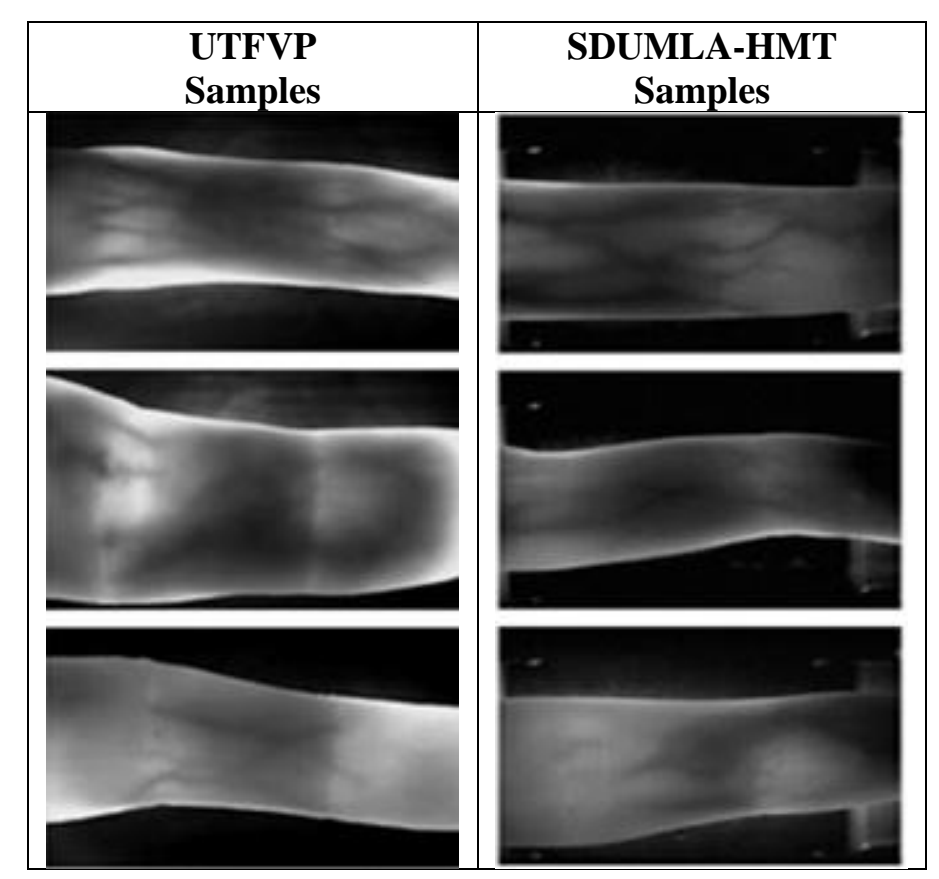

Fig. 5 Samples of finger-vein images taken from UTFVP and SDUMLA-HMT Datasets 


\section{B. Implementation}

In biometric recognition system, most often when handcrafted feature approach is used for feature extraction as in this paper, data enrollment is used instead of training. When a learning machine approach is used, then the system is trained. For data enrollment, the images of both datasets resized and normalized to $(192 \times 64)$ pixels. The strategy of a leave-one-out cross-validation is used. In this strategy, the CLBP components of all the images are enrolled and the three CLBP components of one image at a time is used as a test image against the three CLBP components of all other images in the enrolled data. This means that, the total number of HD measures is $(3 \times 1,036,080)$ for the UTFVP dataset and $(3 \mathrm{x}$ $7,279,020)$ for SDUMLA-HMT dataset. However, a substantial decrease in these numbers may achieve after excluding a portion of the enrolled CLBP components from the HD competition by the use of threshold values for $\mathrm{x}$ and $\mathrm{y}$ displacements. During the implementation, these threshold values were chosen as ( $\mathrm{Tx}=20$ and Ty $=10$ ), where $T x$ is the displacement in the $\mathrm{x}$-direction and $\mathrm{Ty}$ is the displacement in the $\mathrm{y}$-direction.

\section{Results}

The performance of the system, the recognition rate and the Equal Error rate (IRR and EER) was calculated with and without POC and shown in Table 2. The results reveal that the contribution of POC to the performance of the system is significant when used for image alignment with HD measure for matching. Especially for the UTFVP database, this suffers from image misalignment. The IRR and EER values were (99.66\% and $0.139 \%)$ for UTFVP dataset and $(98.95 \%$ and $0.53 \%)$ for SDUMLA-HMT dataset. These results indicate that a significant improvement was achieved in the performance of the system, especially with UTFVP dataset, which suffers from severe misalignment. The IRR and EER values of the system without POC for the UTFVP dataset were (93\% and 3.8\%). For the SDUMLA-HMT dataset, since it does not suffer from a severe image misalignment, the IRR and EER values were still high even without the use of POC $197.27 \%$ and $1.42 \%)$. This result indicates that the performance of CLBP as an image descriptor is high when the displacement between the images is small. The results without POC indicate that the device that was used for creating SDUMLA-HMT dataset guarantees better finger firming compared to that used for creating UTFVP dataset. In order to verify the performance of the system the ROC curves of the system with and without POC are given in Figures 6 and 7 for the UTFVP and SDUMLAHMT datasets.

TABLE 2 Performance of the proposed system with and without POC for UTFVP and SDUMLA-HMT datasets

\begin{tabular}{|c|c|c|c|c|}
\hline \multirow{2}{*}{ Database Type } & \multicolumn{3}{|c|}{ Without POC HD Matching } & \multicolumn{2}{c|}{ POC Alignment HD Matching } \\
& IRR\% & EER\% & IRR\% & EER\% \\
\hline UTFVP & 93 & 3.8 & 99.66 & 0.139 \\
\hline SDUMLA-HMT & 97.27 & 1.43 & 98.95 & 0.53 \\
\hline
\end{tabular}




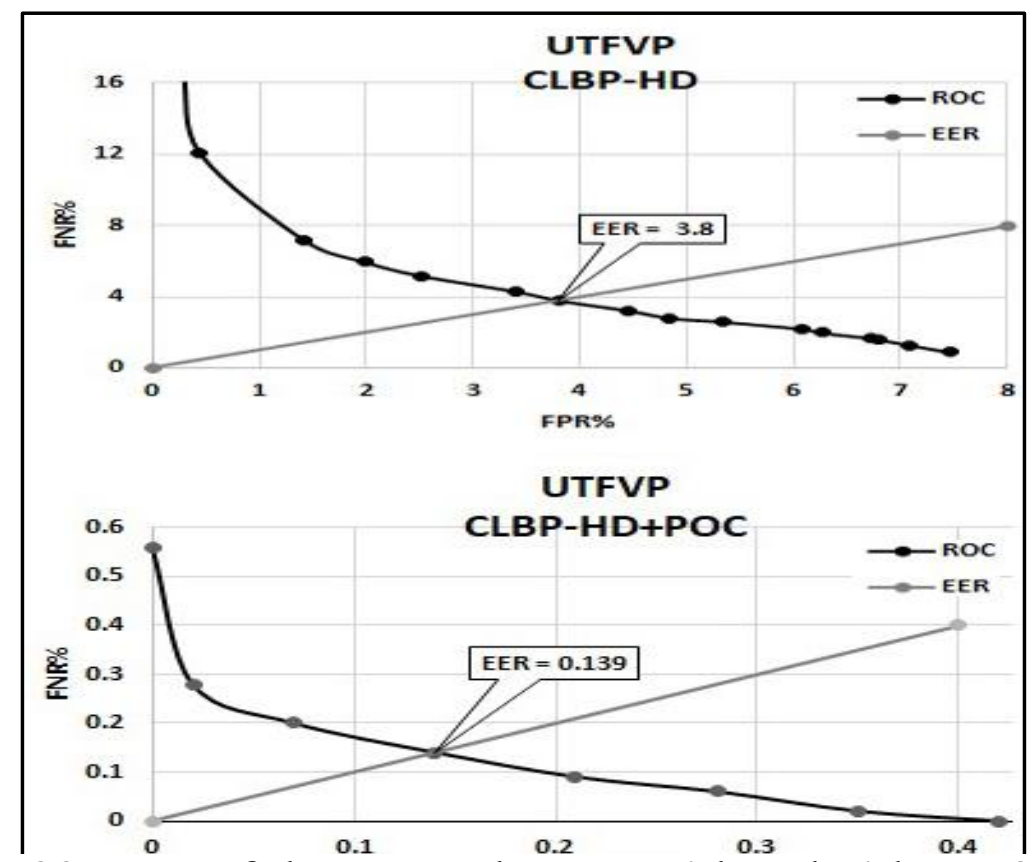

Fig. 6 ROC curves of the proposed system with and without POC using UTFVP dataset

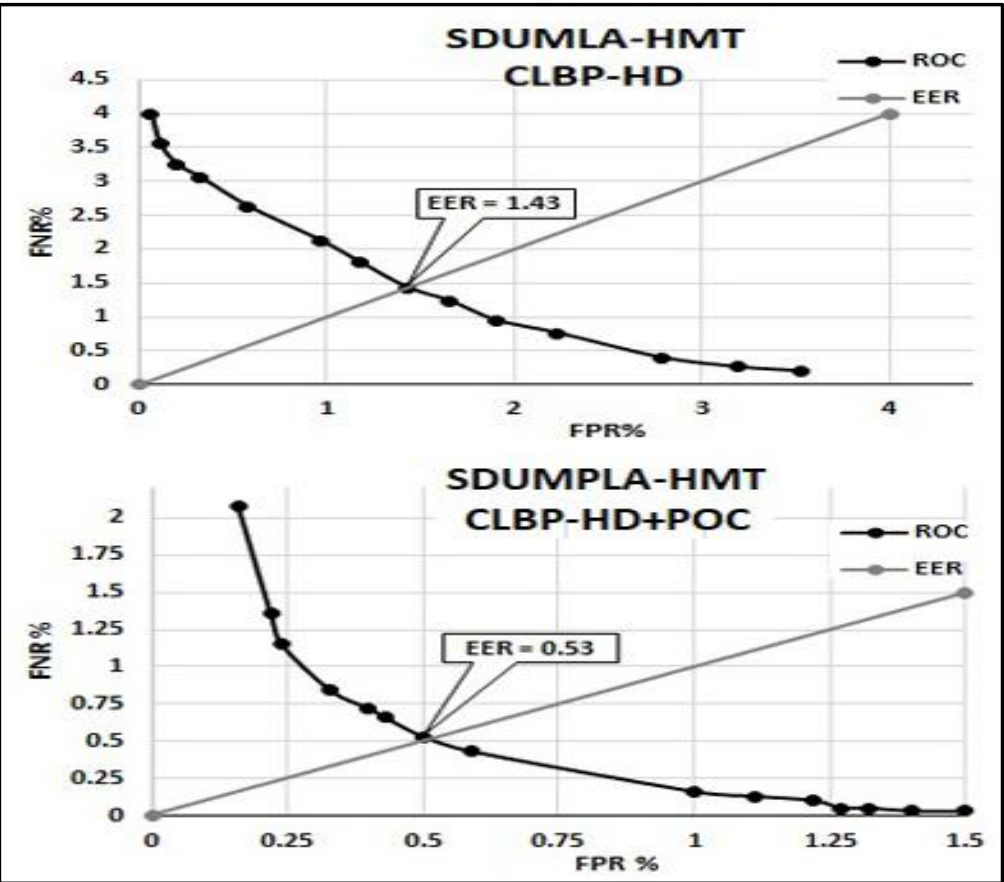

Fig. 7 ROC curves of the proposed system with and without POC using SDUMLA-HMT dataset

\section{SYSTEM EVALUATION}

Two criteria were used to evaluate the system performance, the accuracy and the speed. 


\section{A. Accuracy}

The evaluation of the system is done by comparing its performance to those achieved by previous works for the same datasets but different image descriptors such as such as LBP, RLT, MC, etc., Table 3 and Table 4. However, in most of the previous works one recognition metric is mentioned, either IRR or EER. These two tables show that the proposed system performs better than most of the previous systems for both datasets. This approve the novelty of the proposed system.

TABLE 3 Performance of the proposed system versus the performance of the previous systems using UTFVP Database

\begin{tabular}{|c|c|c|c|}
\hline $\begin{array}{c}\text { Finger-vein } \\
\text { Systems } \\
\text { [Reference] }\end{array}$ & $\begin{array}{c}\text { Feature } \\
\text { Extraction }\end{array}$ & IRR\% & $\begin{array}{c}\text { EER } \\
\%\end{array}$ \\
\hline Kauba et al., 2014 & LBP & - & 6.03 \\
\hline [32] & RLT & - & 7.75 \\
Kauba et al., 2015 & MC & - & 0.47 \\
[51] & WLD & - & 2.00 \\
\hline Xie et al., 2015 [52] & LBP & - & 8.62 \\
\hline Kauba et al., 2016 & RLT & - & 1.54 \\
[53] & MC & - & 0.47 \\
WLD & - & 0.51 \\
\hline Lu et al., 2018 [30] & LBP & - & 7.75 \\
LDP & - & 10.66 \\
Das et al., 2019 [4] & PHDCP & - & 2.91 \\
\hline The proposed & CNN & 95.56 & - \\
\hline system & CLBP & 99.66 & 0.139 \\
\hline
\end{tabular}

TABLE 4 Performance of the proposed system versus the performance of the previous systems using SDUMLA-HMT Database

\begin{tabular}{|c|c|c|c|}
\hline $\begin{array}{c}\text { Finger-vein } \\
\text { Systems } \\
\text { [Reference] }\end{array}$ & $\begin{array}{c}\text { Feature } \\
\text { Extraction }\end{array}$ & $\begin{array}{c}\text { IRR } \\
\%\end{array}$ & $\begin{array}{c}\text { EER } \\
\%\end{array}$ \\
\hline Lu et al., 2013 [24] & LBP & $\begin{array}{c}98.7 \\
9\end{array}$ & 2.84 \\
\hline Shin et al., 2014 [54] & LBP & - & 3.08 \\
\hline $\begin{array}{c}\text { Yahaya et al., 2016 } \\
{[27]}\end{array}$ & MCDF & $\begin{array}{c}99.1 \\
7\end{array}$ & - \\
\hline $\begin{array}{c}\text { Syarif et al., 2017 } \\
{[29]}\end{array}$ & EMC+HOG & - & 0.14 \\
\hline $\begin{array}{c}\text { Meng et al., 2018 } \\
{[31]}\end{array}$ & Dense SIFT & 94 & 2.86 \\
\hline $\begin{array}{c}\text { Wang et al., 2019 } \\
{[32]}\end{array}$ & DCGWLD & $\begin{array}{c}99.4 \\
2\end{array}$ & 0.78 \\
\hline Hong et al., 2017 [3] & CNN & - & 3.9 \\
\hline Das et al., 2019 [4] & CNN & $\begin{array}{c}97.4 \\
8\end{array}$ & - \\
\hline The proposed system & CLBP & 98.95 & 0.53 \\
\hline
\end{tabular}




\section{B. Speed}

The computation times required for the stages of pre-processing and feature extraction (CLBP components) for both datasets was the same $(0.075 \mathrm{sec}$ and $0.275 \mathrm{sec})$, since same image-size $(192 \times 64)$ pixels was used for both datasets. The computation time for the matching stage depends on dataset size; the larger the dataset is the larger is the number of POC and HD measures. The times for one POC and three HD measures are $(0.006 \mathrm{sec}$ and $0.024 \mathrm{sec})$ respectively. The time of HD measure includes the time of measuring HD distance between the enrolled CLBP components and the CLBP components of the test image. The computation times for matching stage for the two datasets when POC is not used are shown in Tables 5 . The details of Table 5 are restricted to one test, because in real practice, when the system is operational, only the computation time of one test is important.

\section{TABLE 5 Computation time of the matching stage for one test without POC} for UTFVP and SDUMLA-HMT datasets

\begin{tabular}{|c|c|c|c|}
\hline $\begin{array}{c}\text { Database } \\
\text { Type }\end{array}$ & $\begin{array}{c}\text { HD single } \\
\text { Measure (sec) }\end{array}$ & $\begin{array}{c}\text { Number of } \\
\text { HD Measures }\end{array}$ & $\begin{array}{c}\text { Matching One } \\
\text { Test (sec) }\end{array}$ \\
\hline UTFVP & 0.024 & 1439 & 34.53 \\
\hline SDUMLA-HMT & 0.024 & 3815 & 91.56 \\
\hline
\end{tabular}

Since the strategy of leave-one-out cross-validation is used, for the UTFVP dataset, (1439) HD measurements are required and for SDUMLA-HMT dataset, (3815) HD measurements are required.

When POC is used, the number of HD measure for the enrolled CLBP components will be decreased, since only a portion of the CLBP components will be excluded initially by the threshold test for the $\mathrm{x}$ and $\mathrm{y}$ displacements. However, the time of calculating POC for all possible pairs within the dataset will be added. This time was $(0.006 \times 1439=8.63 \mathrm{sec})$ for UTFVP dataset and was $(0.006 \times 3815$ $=22.89 \mathrm{sec}$ ) for SDUMLA-HMT dataset. Now, for each test operation, the average number of enrolled images that passed the displacement threshold test was (166 out of 1439) for UTFVP dataset and was (528 out of 3815) for SDUMLA-HMT dataset. This means that, for the UTFVP dataset, only (23\%) of the enrolled images require HD measurements and matching competitions and for the SDUMLA-HMT datasets, only (14\%) of the enrolled images require HD measurements and matching competitions. This average number/test of passed images is measured by dividing the total number of the passed images for all possible tests by the number of images in the dataset. The total number of the passed images for the UTFVP dataset was (239444) and for the SDUMLA-HMT was (2014400). The average number of passed images / test is $(239444 / 1440=166)$ for the UTFVP dataset and $(2014400 / 3816=528)$ for the SDUMLA-HMT dataset. Thus, given the HD measurement time for each three CLBP components with the test image as $(0.024 \mathrm{sec})$, the total time required for one test is $(0.024 \times 166=3.984 \mathrm{sec})$ for the UTFVP dataset and $(0.024 \times 528=12.67 \mathrm{sec})$ for the SDUMLA-HMT dataset. Now, adding the times required by the process of POC for each test, which are $(8.63 \mathrm{sec}$ and $22.89 \mathrm{sec}$ ) for UTFVP and SDUMLA-HMT datasets respectively, the total times for the matching stage for the two datasets, UTFVP and SDUMLA-HMT are (12.61 sec and 35. $56 \mathrm{sec}$ ) respectively. These times are competitive compared to (34.53 sec and $91.56 \mathrm{sec}$ ) of Table 5, when POC is not used. These results lead to the conclusion that, the use of $\mathrm{POC}$ as a priori matching operation decreases the 
computation time of the matching stage if handled properly, in contrast to the common notion that POC increases the computation time of the system.

It should be mentioned that these computation times are subject to the computer specifications used in this work, which were: (Intel(R) Core(TM) i7$6700 \mathrm{HQ}$ CPU @2.60 GHz), windows 10 operating system, x64 - based processor.

TABLE 6 Computation time of the matching stage for one test with POC for UTFVP and SDUMLA-HMT datasets

\begin{tabular}{|c|c|c|c|}
\hline $\begin{array}{c}\text { Database } \\
\text { Type }\end{array}$ & \multicolumn{2}{|c|}{ Time for all } \\
POC (sec) & $\begin{array}{c}\text { Time for HD Measure } \\
\text { Passed CLBP } \\
\text { Components } \\
\text { (sec) }\end{array}$ & $\begin{array}{c}\text { Total Time of } \\
\text { Matching One } \\
\text { Test } \\
\text { (sec) }\end{array}$ \\
\hline UTFVP & 8.63 & 3.984 & 12.61 \\
\hline SDUMLA-HMT & 22.89 & 12.67 & 35.56 \\
\hline
\end{tabular}

\section{CONCLUSIONS}

The proposed system of finger-vein recognition achieved higher IRR and Lower EER values for both databases UTFVP and SDUMLA-HMT compared to those achieved by the previous systems. This is due to some innovative features that have been used in the system, which can be summarized as follows:

1. The use of the image descriptor CLBP, which contains detailed vein features, compared to previous image descriptors.

2. The use of POC technique has contributed significantly to improving the performance of the system. This was evident from the comparison of Table 2 , which revealed the improvement in the IRR and EER after the use of POC, especially for the UTFVP database, which suffers from misalignment.

3. The use of POC technique as a priori matching operation speeds up the system since it excludes a portion of the enrolled images from the process of distance matching competition. The improvement in system speed becomes more significant for large database such as SDUMLA-HMT.

4. The implementation of POC on CLBP components makes the system more secure against any attack on personal information, since the original images are not stored in the enrolled database.

\section{ACKNOWLEDGMENT}

The authors would like to thank the University of Duhok, Kurdistan Region of Iraq for its support. They would also like to express their deep thanks to Shandong University, Jinan, China, the producer of SDUMLA-HMT multimodal biometrics datasets. Also deep thanks go to the University of Twente Finger Vascular Pattern, the producer of UTFVP dataset.

\section{REFERENCES}

[1]. E. C. Lee, H. Jung, and D. Kim,"New finger biometric method using near infrared imaging", Sensors, Vol. 11, 2011, pp. 2319-2333. 
[2]. S. Khellat-Kihel, R. Abrishambaf, N. Cardoso, J. Monteiro, and M. Benyettou, "Finger-vein recognition using Gabor filter and Support Vector Machine", in International Image Processing, Applications and Systems Conference, IPAS 2014, 2014, pp. 1-6.

[3]. H. G. Hong, M. B. Lee, and K. R. Park, "Convolutional Neural NetworkBased Finger-vein Recognition Using NIR Image”, Sensors, Vol. 17, 2017.

[4]. R. Das, E.Piciucco, E.Maiorana, and P.Campisi, "Convolutional Neural Network for Finger-vein based Biometric Identification", in IEEE Transaction for Information Forensics and Security, Vol. 14, No. 2, 2019, pp. 360-373.

[5]. A. AK. Tahir, S.S. Dawood, and S. Anghelus, "An Iris Recognition System Using ANew Method of Iris Localization", International Journal of Open Information Technologies, 2021, In press.

[6]. C. Otti, "Comparison of biometric identification methods", IEEE 11th International Symposium on Applied Computational Intelligence and Informatics (SACI), May 12-14, 2016, Timisoara, Romania, 2016, pp. 339-344.

[7]. K. Malik, and S. Bhattacharya, "Comparative Study of Different Biometric Features", International Journal of Advanced Research in Computer and Communication Engineering, Vol. 2, No. 7, 2013, pp. 2776-2784.

[8]. R. Saini, N. Rana, "Comparison of Various Biometric Methods", International Journal of Advances in Science and Technology (IJAST), Vol. 2, No. 1, 2014, pp. 24-30.

[9]. A.AK. Tahir, and S. Anghelus, "Human Biometrics and Biometric Recognition Systems; An Overview", A XIX-a International Conference on Multidisciplinary, "Professor Dorin Paul - Romanian hydropower founder", Vol. 35/2019, pp. 431-446.

[10]. A. A. Tahir, and S. Anghelus, "An accurate and fast method for eyelid detection", International Journal of Biometrics, Vol. 12, No. 2, 2020, pp. 163-178.

[11]. D. Wang, J. Li, and G. Memik, "User Identification based on Finger-vein Patterns for Consumer Electronics Devices", IEEE Transactions on Consumer Electronics, Vol. 56, No. 2, 2010, pp. 799-804.

[12]. J. Yang, J. Wei, and Y. Shi, "Accurate ROI Localization and Hierarchical Hyper-sphere Model for Finger-vein Recognition", Neurocomputing, 2018.

[13]. N. Miura, A.Nagasaka, and T. Miyatake, "Feature extraction of finger-vein patterns based on repeated line tracking and its application to personal identification", Machine Vision and Applications, Vol. 15, 2004, pp. 194203.

[14]. N. Miura, A.Nagasaka, and T. Miyatake, "Extraction of finger-vein patterns using maximum curvature points in image profiles", IEICE Transactions on Information and Systems, Vol. 90, No. 8, 2007, pp. 1185-1194.

[15]. W. Song, T. Kim, H. C. Kim, K. R. PARK,"A finger-vein verification system using mean curvature", Pattern Recognition Letters, Vol. 32, 2011, pp. 1541-1647.

[16]. E.C. Lee, H. C. Lee, and K. P. Park, "Finger-vein Recognition Using Minutia-Based Alignment and Local Binary Pattern-Based Feature Extraction", International Journal of Imaging Systems and Technology, Vol. 9, No. 3, 2009, pp. 179-186.

[17]. H.C. Lee, B.J. Kang, E.C. Lee, and K. R. Park, "Finger-vein recognition using weighted local binary pattern code based on a support vector machine", Journal of Zhejiang University SCIENCE C (Computer \& Electronics), Vol. 11, No. 7, 2010, pp. 514-524. 
[18]. K. R. Park, "Finger-vein Recognition By Combining Global And Local Features Based On SVM", Computing and Informatics, Vol. 30, No. 2, 2011, pp. 295-309.

[19]. B. A. Rosdi, W.S. Chai, and A. A. Shahrel, "Finger-vein Recognition Using Local Line Binary Pattern", Sensors, Vol. 11, 2011, pp. 11357-11371.

[20]. B. Huang, Y. Dai, R. Li, D. Tang, and W. Li, "Finger-vein Authentication Based On Wide Line Detector And Pattern Normalization", in Proceedings International Conference on Pattern Recognition, 2010, pp. 1269-1272.

[21]. Y. Lu, S. J. Xie, S.Yoon, D. S. Park, "Finger-vein Identification Using Polydirectional Local Line Binary Pattern", International Conference on ICT Convergence (ICTC), 2013, pp. 61-65.

[22]. H. Liu, L. Song, G. Yang, L. Yang, and Y. Yin, "Customized Local Line Binary Pattern Method for Finger-vein Recognition", in Lecture Notes in Computer Science (including subseries Lecture Notes in Artificial Intelligence and Lecture Notes in Bioinformatics), Springer US, 2017, pp. 314-323.

[23]. J. F. Yang, J. L. Yang, Y. H. Shi, "Finger-vein recognition based on a bank of Gabor filters", In: Proceedings of ACCV'09, 2009, pp. 374-383.

[24]. Y. Lu, S. Yoon, and D. S. Park, "Finger-vein Recognition based on Matching Score-Level Fusion of Gabor Features", The Journal of Korean Institute of Communications and Information Sciences, Vol. 38A, No. 2, 2013, pp. 174-182.

[25]. J. Peng, Q. Li, N. Wang, A. A. Abd El-Latif, and X. Niu, "An Effective Preprocessing Method for Finger-vein Recognition", Fifth International Conference on Digital Image Processing (ICDIP 2013), 2013, edited by Yulin Wang, Xie Yi, Proc. of SPIE, Vol. 8878.

[26]. M. Kaur, G. Babbar, and C. E. C.Landran, "Finger-vein Detection using Repeated Line Tracking, Even Gabor and Multilinear Discriminant Analysis (MDA )', Vol. 6, No. 4, 2015, pp. 3280-3284.

[27]. Y. H. Yahaya, S. M. Shamsuddin, and W. Y. Leng, "Finger-vein Feature Extraction Using Discretization", 4th International Conference on Artificial Intelligence and Computer Science, 2016.

[28]. S. Brindha, "Finger-vein recognition", International Research Journal of Engineering and Technology (IRJET), Vol. 4, No. 4, 2017, pp. 1298-1300.

[29]. M. A. Syarif, T. S. Ong, A. B. J. Teoh, and C. Tee, "Enhanced Maximum Curvature Descriptors for Finger-vein Verification", Multimedia Tools and Applications, Vol. 76, No. 5, 2017, pp. 6859-6887.

[30]. Y. Lu, S. Yoon, S. Wu, and D. S. Park, "Pyramid Histogram of Double Competitive Pattern for Finger-vein Recognition", IEEE Acess, Vol. 6, 2018, pp. 56445-56456.

[31]. X. Meng, X. Xi, G. Yang, et al., "Finger-vein recognition based on deformation information", SCIENCE CHINA, Information Sciences, Vol. 61, 2018.

[32]. H. Wang, M. Du, J. Zhou, and L. Tao, "Weber Local Descriptors with Variable Curvature Gabor Filter for Finger-vein Recognition”, IEEE Acess, Vol. 7, 2019, pp. 108261-108277.

[33]. S. Tang, S. Zhou, W. Kang, Q. Wu, and F. Deng, "Finger-vein Verification using a Siamese Convolutional Neural Network", IET Biometrics, 2019.

[34]. A. I. Mohammed, and A. AK. Tahir, A. AK., "A New Image Classification System Using Deep Convolution Neural Network And Modified Amsgrad Optimizer", Journal of University of Duhok (Pure and Eng. Science), Vol. 22, No.2, 2019, pp. 89-101.

[35]. A. I. Mohammed, and A. AK. Tahir, "A New Optimizer for Image Classification using Wide ResNet (WRN)", Academic Journal of Nawroz University, Vol. 9, No. 4, 2020, pp. 1-13. 
[36]. C. Kauba, J.Reissig, J. and A. Uhl, "Pre-processing cascades and fusion in finger-vein recognition", In Proceedings of the International Conference of the Biometrics Special Interest Group, 2014, pp. 99-110.

[37]. K. Q. Wang, A. S.Krisa, X. Q. Wu, and Q. S. Zhao, "Finger vein recognition using LBP variance with global matching", Proceedings of the International Conference on Wavelet Analysis and Pattern Recognition, 2012, pp. 196200.

[38]. A. A. Mustafa, and A. AK. Tahir, "Improving the Performance of Finger vein Recognition System Using A New Scheme of Modified Preprocessing Methods", Academic Journal of Nawroz University, Vol. 9, No. 3, 2020, pp. 397- 409.

[39]. Z. Guo, Z. Lei, and Z. David, "A Completed Modeling of Local Binary Pattern Operator for Texture Classification", IEEE Transactions on Image Processing, Vol. 19, No. 6, 2010, pp. 1657-1663.

[40]. Y. Zhao, D. Huang, and W. Jia, "Completed Local Binary Count for Rotation Invariant Texture Classification", IEEE Transactions On Image Processing, Vol. 21, No. 10, 2012, pp, 4492-4497.

[41]. T. H. Rassem and B. E. Khoo, "Completed Local Ternary Pattern for Rotation Invariant Texture Classification", Hindawi Publishing Corporation, The Scientific World Journal, Volume 2014, Article ID 373254, pp.1-10.

[42]. M. Guermoui, and M. L. Mekhalfi, "A Sparse Representation of Complete Local Binary Pattern Histogram for Human Face Recognition", 2016, pp.14.

[43]. S. NagarajaC. J. Prabhakar and P. U. Kumar, "Complete Local Binary Pattern for Representation of Facial Expression Based on Curvelet Transform", Proceeding of International Conference on Multimedia Processing, Communication \& Info. Tech., MPCIT, 2013, Association of Computer Electronics and Electrical Engineers, E, DOI: 03.AETS.2013.4.32.

[44]. K. Takita, T. Aoki, Y. Sasaki, T. Higuchi, and K. Kobayashi, "High Accuracy Subpixel image registration based on Phase-Only-Correlation", IEICE Transaction on Fundamentals, Vol. E86-A, No. 8, 2003, pp. 1925-1934.

[45]. K. Ito, H. Nakajima, K. Kobayashi, T. Aoki, and T. Higuchi,"A Fingerprint Matching Algorithm Using Phase Only Correlation", IEICE Transaction on Fundamentals, Vol. E87-A, No. 3, 2004, pp. 682-691.

[46]. K. Ito, T. Aoki, H. Nakajima, K. Kobayashi, and T. Higuchi, "A Palmprint Recognition Algorithm Using Phase Only Correlation", IEICE Transaction on Fundamentals, Vol. E91-A, No. 4, 2008, pp. 1023-1030.

[47]. M. Miura, S. Sakai, S. Aoyama, et al., "High-Accuracy Image Matching Using Phase-Only Correlation and Its Application", SICE Annual Conference, 2012, pp. 307-312.

[48]. M. Vanoni, P. Tome, L. El Shafey, and S. Marcel, "Cross-Database Evaluation Using an Open Finger-vein Sensor", IEEE Workshop on Biometric Measurements and Systems for Security and Medical Applications (BIOMS) Proceedings, Rome, 2014, pp. 30-35.

[49]. Y. Yin L. Liu and X. Sun, "SDUMLA-HMT: A multimodal Biometric Database", In Biometric Recognition by (Sun, Z., L., J., Chen, X., Tan, T. (Eds.)), Springer Berlin Heidelberg, 2011, pp. 260-268.

[50]. Y. Lu, S. J.Xie, S. Yoon, Z. Wang, and D. S. Park, "An Available Database for the Research of Finger-vein Recognition", the 6th International Congress on Image and Signal Processing, 2013, pp. 410-415.

[51]. C. Kauba, and A. Uhl, "Sensor Ageing Impact On Finger-vein Recognition", International Conference on Biometrics (ICB), Phuket, 2015, pp. 113-120. 
[52]. S. J. Xie, Y. Lu, S. Yoon, J. Yang, and D. S> Park, "Intensity Variation Normalization for Finger-vein Recognition Using Guided Filter Based Singe Scale Retinex", Sensors, Vol. 15, 2015, pp. 17089-17105.

[53]. C. Kauba, A. Uhl, E.Piciucco, E. Maiorana, and P. Campisi, "Advanced variants of feature level fusion for finger-vein recognition", In the International Conference of the Biometrics Special Interest Group (BIOSIG), 2016, pp. 1-7.

[54]. K. Y. Shin, Y. H. Park, D. T. Nguyen, and K. R. Park, "Finger-vein Image Enhancement Using a Fuzzy-Based Fusion Method with Gabor and Retinex Filtering", Sensors, Vol. 14, 2014, pp. 3095-3129. 